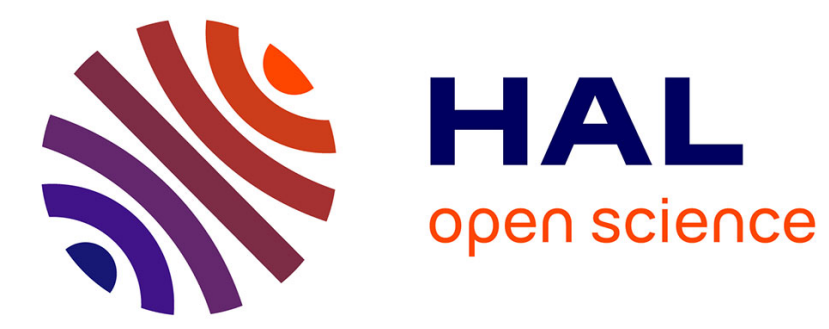

\title{
Temperature dependence of spin exchange frequency shifts in $\mathbf{H}-\mathbf{H}$ collisions
}

\author{
M. Desaintfuscien, J. Viennet, C. Audouin, J. Vanier
}

\section{To cite this version:}

M. Desaintfuscien, J. Viennet, C. Audouin, J. Vanier. Temperature dependence of spin exchange frequency shifts in $\mathrm{H}-\mathrm{H}$ collisions. Journal de Physique Lettres, 1975, 36 (12), pp.281-284. 10.1051/jphyslet:019750036012028100 . jpa-00231209

\section{HAL Id: jpa-00231209 https://hal.science/jpa-00231209}

Submitted on 1 Jan 1975

HAL is a multi-disciplinary open access archive for the deposit and dissemination of scientific research documents, whether they are published or not. The documents may come from teaching and research institutions in France or abroad, or from public or private research centers.
L'archive ouverte pluridisciplinaire HAL, est destinée au dépôt et à la diffusion de documents scientifiques de niveau recherche, publiés ou non, émanant des établissements d'enseignement et de recherche français ou étrangers, des laboratoires publics ou privés. 


\title{
TEMPERATURE DEPENDENCE OF SPIN EXCHANGE FREQUENCY SHIFTS IN H-H COLLISIONS
}

\author{
M. DESAINTFUSCIEN, J. VIENNET and C. AUDOUIN
}

Laboratoire de l'Horloge Atomique (*), Université Paris-Sud, Bât. 221, 91405 Orsay, France

\section{J. VANIER $(* *)$}

Laboratoire d'Electronique Quantique, Faculté des Sciences, Université Laval, Québec, Canada

(Reçu le 8 juillet 1975, accepté le 15 septembre 1975)

\begin{abstract}
Résumé. - La mesure du déplacement de la fréquence de transition hyperfine, par échange de spins, dans les collisions hydrogène-hydrogène est décrite dans la gamme de température $77 \mathrm{~K}-363 \mathrm{~K}$.

Les données expérimentales s'accordent avec les résultats d'un calcul théorique de la variation avec la température de la section efficace du déplacement de fréquence par échange de spins et avec un résultat expérimental obtenu à $308 \mathrm{~K}$, à l'aide d'une technique différente. Nous confirmons expérimentalement que la section efficace du déplacement de fréquence par échange de spins change de signe à basse température, comme le prévoit la théorie.
\end{abstract}

\begin{abstract}
Experimental data on the spin exchange frequency shifts due to hydrogen-hydrogen spin exchange collisions are reported over the temperature range $77 \mathrm{~K}$ to $363 \mathrm{~K}$. They are found to agree with the results of a theoretical calculation of the temperature dependence of the spin exchange frequency shift cross-section and with an experimental result obtained by a different technique near room temperature.

Our experimental results confirm the prediction that the spin exchange frequency shift crosssection changes sign at low temperature.
\end{abstract}

1. Introduction. - It is known, from the work of Bender [1], Balling et al. [2] and Crampton [3, 4] that spin-exchange collisions between hydrogen atoms introduce a frequency shift, as well as a broadening $[5,6]$ in the ground state hyperfine line.

This angular frequency shift can be conveniently written as follows [4], [7]

$$
\omega_{0}^{\prime}-\omega_{0}=-\frac{\lambda}{4} n \bar{v}_{\mathrm{r}}\left(\rho_{22}-\rho_{44}\right)+\varepsilon_{\mathrm{H}} n \bar{v}_{\mathrm{r}} \sigma
$$

where $\omega_{0}$ is the angular frequency for the $\Delta F=1$, $\Delta m_{F}=0$ transition corresponding to the absence of hydrogen-hydrogen collisions;

$\omega_{0}^{\prime}$ is the spin-exchange shifted angular frequency;

$\lambda$ is the spin-exchange frequency shift cross-section; $n$ is the density of hydrogen atoms ;

$\bar{v}_{\mathrm{r}}$ is the mean relative velocity of the atoms ;

$\left(\rho_{22}-\rho_{44}\right)$ is the population difference between the $F=1, m_{F}=0$ and $F=0, m_{F}=0$ hyperfine levels, and

$\sigma$ is the spin exchange cross-section.

(*) Equipe de Recherche du C. N. R. S.

$\left({ }^{* *}\right)$ On leave at the Laboratoire de l'Horloge Atomique, Université Paris-Sud.
$\varepsilon_{\mathrm{H}}$ is a recently introduced parameter [4], [8] which represents the effect of the interruption of the oscillating magnetic moments during collisions.

The value of the parameter $\lambda$ has been calculated by Allison [9] using a specific model for the hydrogenhydrogen interaction potentials [10] and it was then theoretically found that, while positive at room temperature, the value of $\lambda$ becomes negative at low temperature, below about $205 \mathrm{~K}$.

The purpose of this paper is to report the results of an experimental determination of the temperature dependence of the spin exchange frequency shifts, from measurements made with a hydrogen maser [11]. Taking into account the temperature dependence of $\varepsilon_{\mathrm{H}}$, as evaluated by Crampton [4] $\left({ }^{1}\right)$, it is confirmed that $\lambda$ changes sign at low temperature.

2. Principle of the measurement. - The hydrogen maser output frequency $\omega$, including the effects of hydrogen-hydrogen collisions, is given by [4], [7]

$$
\omega-\omega_{0}=\frac{1}{T_{2}}\left(\frac{2 \Delta \omega_{\mathrm{c}}}{\delta \omega_{\mathrm{c}}}-C \lambda\right)+\frac{\varepsilon_{\mathrm{H}}}{T_{\mathrm{H}}}
$$

(1) Crampton, S. B., Private communication 
with, in C.G.S. units,

$$
C=\bar{v}_{\mathrm{r}} V_{\mathrm{c}} \hbar / 16 \pi Q \eta V_{\mathrm{b}} \mu_{0}^{2}
$$

where $\omega_{0}$ is the above defined angular frequency of the O-O hyperfine transition, including wall shift, second order Doppler shift and magnetic field bias; $\Delta \omega_{c}$ is the amount of detuning of the cavity from $\omega_{0}$; $\delta \omega_{\mathrm{c}}$ is the width of the cavity resonance;

$h$ is Planck constant, $\hbar=\frac{h}{2 \pi}$;

$\mu_{0}$ is Bohr magneton;

$V_{\mathrm{c}}$ and $V_{\mathrm{b}}$ are respectively, the volume of the cavity and the storage bulb;

$\eta$ is the filling factor;

$Q$ is the cavity quality factor;

$T_{2}$ is the transverse relaxation time of the atomic transition and

$T_{\mathrm{H}}^{-1}=n \bar{v}_{\mathrm{r}} \sigma$ is the part of the longitudinal relaxation rate due to $\mathrm{H}-\mathrm{H}$ collisions.

From expression (2) it can easily be shown that a proper cavity detuning exists for which the maser oscillation frequency becomes independent of the atomic hydrogen density. This was verified experimentally [12] and justifies the usual method of cavity tuning [13]. This method gives the maser oscillation angular frequency the value $\omega_{\mathrm{t}}$ which is defined by [4] :

$$
\omega_{\mathrm{t}}-\omega_{0}=-2 \varepsilon_{\mathrm{H}}\left(\frac{1}{T_{2}}-\frac{1}{2 T_{\mathrm{H}}}\right)=-2 \frac{\varepsilon_{\mathrm{H}}}{T_{2}^{\prime}}
$$

where $\left(T_{2}^{\prime}\right)^{-1}$ represents that part of the transverse relaxation rate which is not due to $\mathrm{H}-\mathrm{H}$ collisions.

The theoretical expression for the frequency dependence of the output power is obtained from results previously developed, to describe the behaviour of the hydrogen maser [14]. One obtains

$$
b^{2}=b_{\max }^{2}-\frac{T_{2}}{T_{1}}\left(\omega-\omega_{0}^{\prime}\right)^{2}
$$

where $b=\mu_{0}\left\langle H_{z}\right\rangle / \hbar$. Here $\left\langle H_{z}\right\rangle$ is the mean value, of the $z$-component of the microwave field amplitude, taken over the volume of the bulb, and $T_{1}$ is the longitudinal relaxation time.

However, one has to be careful since the atomic transition frequency $\omega_{0}^{\prime}$ is not unique, but is a function of the oscillation level, via the population difference $\left(\rho_{22}-\rho_{44}\right)$, as is evident in expression (1). The result is that the relation (5) between power and frequency is represented by a curve which differs, but only slightly, from a parabola. It can easily be shown that the maximum power is obtained at the angular frequency $\omega_{\max }$ given by :

$$
\omega_{\max }-\omega_{0}=-\frac{C \lambda}{T_{2}}+\frac{\varepsilon_{\mathrm{H}}}{T_{\mathbf{H}}} .
$$

A method for the measurement of this frequency consists in measuring the hydrogen maser output level and the oscillation frequency $\omega$, for various cavity detunings [15], and searching for the frequency which gives the maximum output power.

The difference between the angular frequencies of interest, $\omega_{\mathrm{t}}$ and $\omega_{\max }$ equals :

$\omega_{\max }-\omega_{\mathrm{t}}=-C\left(\lambda-2 \varepsilon_{\mathrm{H}} / C\right) / T_{2}=-C \lambda^{*} / T_{2}$

where $\lambda^{*}$ is introduced to represent the total effect of the $\mathrm{H}-\mathrm{H}$ collisions.

3. Experimental results. - We have experimentally determined the frequency difference $\omega_{\max }-\omega_{\mathrm{t}}$ from $77 \mathrm{~K}$ to $363 \mathrm{~K}$ with a hydrogen maser specifically designed to operate over a very large temperature range [16]. In this maser, the microwave cavity and the storage bulb are surrounded by a cryostat made of copper. The cryostat is filled with liquid nitrogen for operation at $77 \mathrm{~K}$. Appropriate temperature regulated liquids (isopentane below room temperature and glycol above that temperature) are smoothly circulated to stabilize the temperature in the range from $130 \mathrm{~K}$ to $363 \mathrm{~K}$. The design allows a temperature homogeneity over the bulb and the microwave cavity better than $0.1 \mathrm{~K}$. Due to the very large thermal inertia of the device, temperature fluctuations at a given point in the microwave cavity are smaller than $0.01 \mathrm{~K}$. The static magnetic field applied to the hydrogen atoms is large enough to make the effects of any magnetic field inhomogeneity negligible. This point was checked using a method described elsewhere [17].

For each temperature of measurement, the cavity resonant frequency was varied by adjusting the position of the central part of one of the end plates of the cavity.

The frequencies were measured by using, as a reference, another hydrogen maser showing fractional frequency fluctuations smaller than $10^{-14}$ over the times of measurement [18].

The experimental procedure consisted of tuning the maser by the classical spin exchange broadening method, and at the same time measuring the level of oscillation with the help of a superheterodyne receiver.

The relaxation times $T_{1}$ and $T_{2}$ were measured [17] as well as the cavity quality factor $Q$ for each temperature considered. The filling factor $\eta[11,13]$ was calculated from the geometry of the storage bulb and cavity.

In the method of tuning through the maximum of output power, the range of the data extended over at least 15 points of the curve and power changes in excess of $6 \mathrm{~dB}$. These experimental data were reduced by computer.

Several methods have been used to determine the values of $\lambda$. In the one described below, the experimental data are corrected in order to fit a parabola. For that purpose, the angular frequency $\omega_{1}$ is introduced, defined as follows :

$$
\omega_{1}=\omega-\left(\omega_{0}^{\prime}-\omega_{\text {max }}\right)
$$


where $\omega_{0}^{\prime}-\omega_{\max }$ is given by :

$$
\omega_{0}^{\prime}-\omega_{\max }=-C \lambda T_{2}\left(\omega_{0}^{\prime}-\omega\right)^{2} .
$$

Eq. (5) can then be written as

$$
b^{2}=b_{\max }^{2}-\frac{T_{2}}{T_{1}}\left(\omega_{\max }-\omega_{1}\right)^{2}
$$

where $\omega_{\max }$ is a constant.

Assuming a given value of $\lambda^{*}$, a parabola is least square fitted to the data (output levels and angular frequencies $\left.\omega_{1}\right)$. The top of the parabola provides a new value of $\lambda^{*}$, using eq. (7), which is then introduced in the same calculation. The process is convergent and gives the values of $\lambda^{*}$ shown in figure $1 a$. The fractional spacing between the corrected experimental data and the parabola obtained at the end of the iterative process is smaller than $1 \%$.

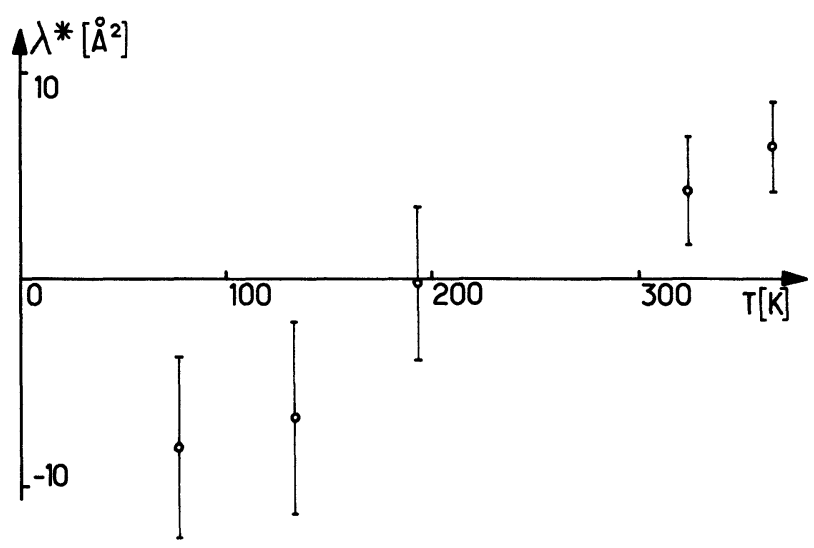

FIG. 1a. - Temperature dependence of the cross-section $\lambda^{*}$ defined in expression (7).

This method does not require a knowledge of the absolute value of $b^{2}$, but only of the relative output power, in arbitrary units. The symmetry of the parabola representing eq. (10) minimizes the errors which would result from residual non-linearities of the receiver. In fact, a simulated non-linearity 10 times larger than the measured one introduces quite a negligible error in the determination of $\left(\omega_{\max }-\omega_{t}\right)$, and therefore of $\lambda^{*}$.

We have also checked that the residual mistuning of the receiver gives a systematic error smaller than $2 \times 10^{-3} \mathrm{rad} . \mathrm{s}^{-1}$ in the measured value of $\left(\omega_{\max }-\omega_{\mathrm{t}}\right)$.

Variation of the cavity quality factor while displacing the plunger would cause a shift in the frequency $\omega_{\max }$. However fractional variations in the cavity quality factor of $10^{-3}$ are very unlikely under the given experimental conditions, and would introduce an additional error smaller than $3 \times 10^{-3} \mathrm{rad}_{\mathrm{s}} \mathrm{s}^{-1}$ on the value of $\left(\omega_{\max }-\omega_{t}\right)$.

The uncertainty due to random errors in the measurement of the angular frequency difference $\omega_{\max }-\omega_{t}$ is evaluated in the following manner. Firstly a parabola

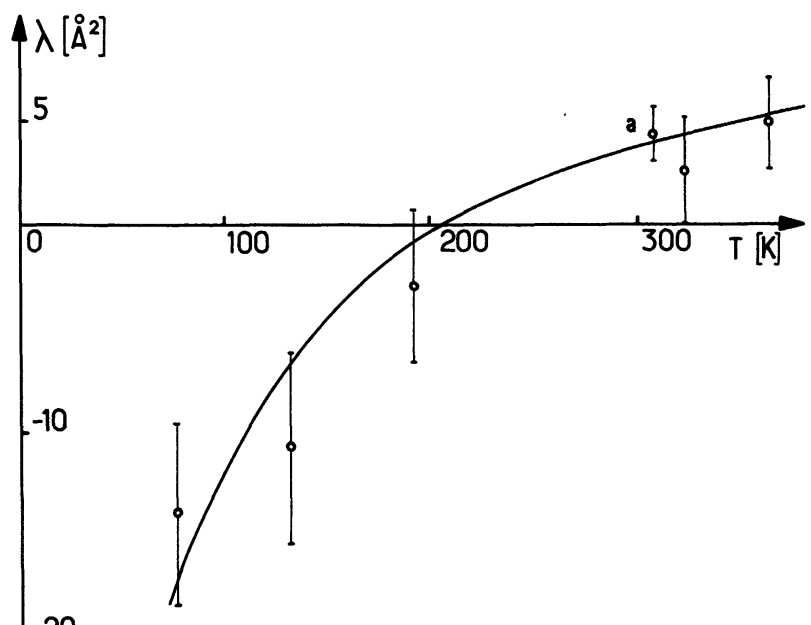

$-20$

FIG. 1b. - Temperature dependence of the $\mathrm{H}-\mathrm{H}$ spin exchange frequency shift cross-section $\lambda$. The points are derived from the experimental data described in this paper, the solid line is that calculated in reference [9] and the point marked $" a \|$ is from reference [8].

is calculated from all the experimental data. Then, the points situated on the left side of this curve are grouped together and used to determine a curve, while the points situated on the right are similarly grouped and used to determine another curve. The difference between the abscissa of the maximum of each of these and the $\omega_{\max }$ of the first parabola passing through all the points gives approximatively the precision to which we can determine the true value of $\omega_{\max }$. The precision in the determination of $\omega_{\mathrm{t}}$ is estimated to be the same as for $\omega_{\max }$. The resulting uncertainty in the measurement of $\left(\omega_{\max }-\omega_{t}\right)$ then amounts to $5 \times 10^{-3} \mathrm{rad} . \mathrm{s}^{-1}$.

Considering all the above mentioned sources of error, the total uncertainty in the value of $\left(\omega_{\max }-\omega_{t}\right)$ equals $6.2 \times 10^{-3} \mathrm{rad} . \mathrm{s}^{-1}$, which determines the precision of the measurement of $\lambda^{*}$. It is worth noticing that, the value of the hyperfine frequency being close to $1420 \mathrm{MHz}$, the quoted uncertainty corresponds to a fractional precision of about $7 \times 10^{-13}$.

4. Comments on the results. - The determination of $\lambda$ from the obtained values of $\lambda^{*}$ requires a knowledge of $\varepsilon_{\mathrm{H}}$. At this time an experimental value of this parameter is available only at $308 \mathrm{~K}[4,8]$, its value being $\varepsilon_{\mathrm{H}}=(-4.04 \pm 0.35) \times 10^{-4}$. For other temperatures, one can use the theoretical result [4] that $\varepsilon_{\mathrm{H}}$ varies in direct proportion to the duration of the collision, and therefore as the inverse of the square root of the temperature.

The obtained values of $\lambda$ are shown in figure $1 b$. The result of the Allison calculations [9] is shown as a solid line and the experimental point marked " $\mathrm{a}$ » refers to the value published by Crampton et al. [8] for a temperature of $308 \mathrm{~K}$ and obtained by a different technique.

The agreement between the theoretical and the 
experimental temperature dependence of the spinexchange cross-section is satisfactory and it is experimentally confirmed that $\lambda$ changes sign at a temperature around $205 \mathrm{~K}$.
Acknowledgments. - We are grateful to Dr. Petit for the design and preparation of the experimental apparatus and also to Pr. S. B. Crampton for helpful comments and suggestions on this work.

\section{References}

[1] BENDER, P. L., Phys. Rev. 132 (1963) 2154.

[2] Balling, L. C., Hanson, R. J. and Pipkin, F. M., Phys. Rev. A 133 (1964) 607.

[3] Crampton, S. B., Ph. D. Thesis, Harvard (1964). Unpublished.

[4] Crampton, S. B. and Wang, H. T. M., Bull. Am. Phys. Soc. 18 (1973) 709 and Crampton, S. B. and Wang, H. T. M., Proceedings of the 28th Annual Symposium on Frequency Control (1974) p. 355

[5] Purcell, E. M. and Fields, G. B., Astrophys. J. 124 (1956) 542.

[6] Wittke, J. P. and Dicke, R. H., Phys. Rev. 103 (1956) 620.

[7] Crampton, S. B., Phys. Rev. 158 (1967) 57.

[8] Crampton, S. B., Duvivier, J. A., Read, G. S. and Williams, E. R., Phys. Rev. A 5 (1972) 1752.

[9] Allison, A. C., Phys. Rev. A 5 (1972) 2695.

[10] Kolos, W. and Wolniewicz, L., J. Chem. Phys. 43 (1965) 2429 .
[11] KlePPNER, D., Goldenberg, H. M. and RamSey, N. F., Phys. Rev. 126 (1962) 603.

[12] Vanier, J. and Vessot, R. F. C., Appl. Phys. Lett. 4 (1964) 122.

[13] Kleppner, D., Berg, H. C., Crampton, S. B., Ramsey, N. F., Vessot, R. F. C., Peters, H. E. and Vanier, J., Phys. Rev. 138 (1965) A 972.

[14] Audoin, C., Revue Phys. Appl. 2 (1967) 309.

[15] Essen, L., Donaldson, R. W., Hope, E. G. and Bangham, M. J., Metrologia 9 (1973) 128.

[16] Desaintfuscien, M., Petit, P., Audoin, C. and Schermann, J. P., C. R. Hebd. Séan. Acad. Sci. B 270 (1970) 906.

[17] Desaintfuscien, M. and Audoin, C., J. Physique 35 (1974) 829.

[18] Petit, P., Viennet, J., Barillet, R., Desaintfuscien, M. and Audorn, C., Metrologia 10 (1974) 61. 